\section{Effektive Therapie von Ohrenschmerzen}

— Ohrenschmerzen sind eines der häufigsten Probleme mit dem Kinder in der HNOPraxis vorgestellt werden. Die physiologischen Bedingungen im Kindesalter - gehäufte Infekte der oberen Luftwege, kurze und horizontal verlaufende Paukenröhre und daraus resultierend eine schlechte Belüftung des Mittelohrs - begünstigen akute Infektionen der Paukenhöhle (akute Otitis media, AOM). Als Sonderform der Mittelohrentzündung kann eine chronisch suppurative Otitis media (CSOM) auftreten, bei der eine chronische Entzündung mit typischen Keimen (Pseudomonas aeruginosa, Staphylococcus aureus) bei zentral perforiertem Trommelfell persistiert. Entzündungen des Außenohrs treten dagegen gehäuft bei verstärktem Wasserkontakt in der Badesaison („Badeotitis“) oder durch unsachgemäße Verhaltensweisen (Reinigung des Gehörgangs mit Wattestäbchen, Fremdkörperinsertion) auf.

Während sich bei der Therapie der AOM das Konzept des „Watchful Waiting" etabliert hat, sollte bei der Otitis externa eine rasche Intervention im Vordergrund stehen. Neben der Reinigung des Gehörgangs kommt dabei der Beseitigung der Erreger und abschwellenden
Maßnahmen besondere Bedeutung zu. Die entsprechenden HNO-Leitlinien empfehlen zur Beseitigung der häufigsten Erreger Pseudomonas aeruginosa, Staphylococcus aureus und Proteus mirabilis eine antibiotische Lokaltherapie. Bewährt hat sich dabei der Wirkstoff Ciprofloxacin. Mit Panotile ${ }^{\circledR}$ Cipro Ohrentropfen steht ein Präparat mit lipophilen und viskösen Eigenschaften (gute Haftung und Benetzung) und einem gut ans Ohrmilieu angepasstem pH von 5,3-6,3 zur Verfügung. Es enthält zudem den Hilfstoff Glycerol,der für seine abschwellenden Eigenschaften bekannt ist. Als einzige Ciprofloxacin-haltige Ohrentropfen kommt Panotile ${ }^{\circledR}$ Cipro dank Einmaldosisbehältnissen ohne potentiell ototoxische Konservierungsstoffe aus. Die hohe Therapiesicherheit spiegelt sich auch in der Tatsache wider, dass Panotile ${ }^{\circledR}$ Cipro als einziges Präparat auch zur leitliniengerechten lokalen Therapie der chronisch eitrigen Otitis media mit Trommelfelldefekt zugelassen ist. Panotile ${ }^{\oplus}$ Cipro kann auch bei liegenden Paukenröhrchen angewendet werden.

gz

Nach Informationen von Pierre Fabre Pharma

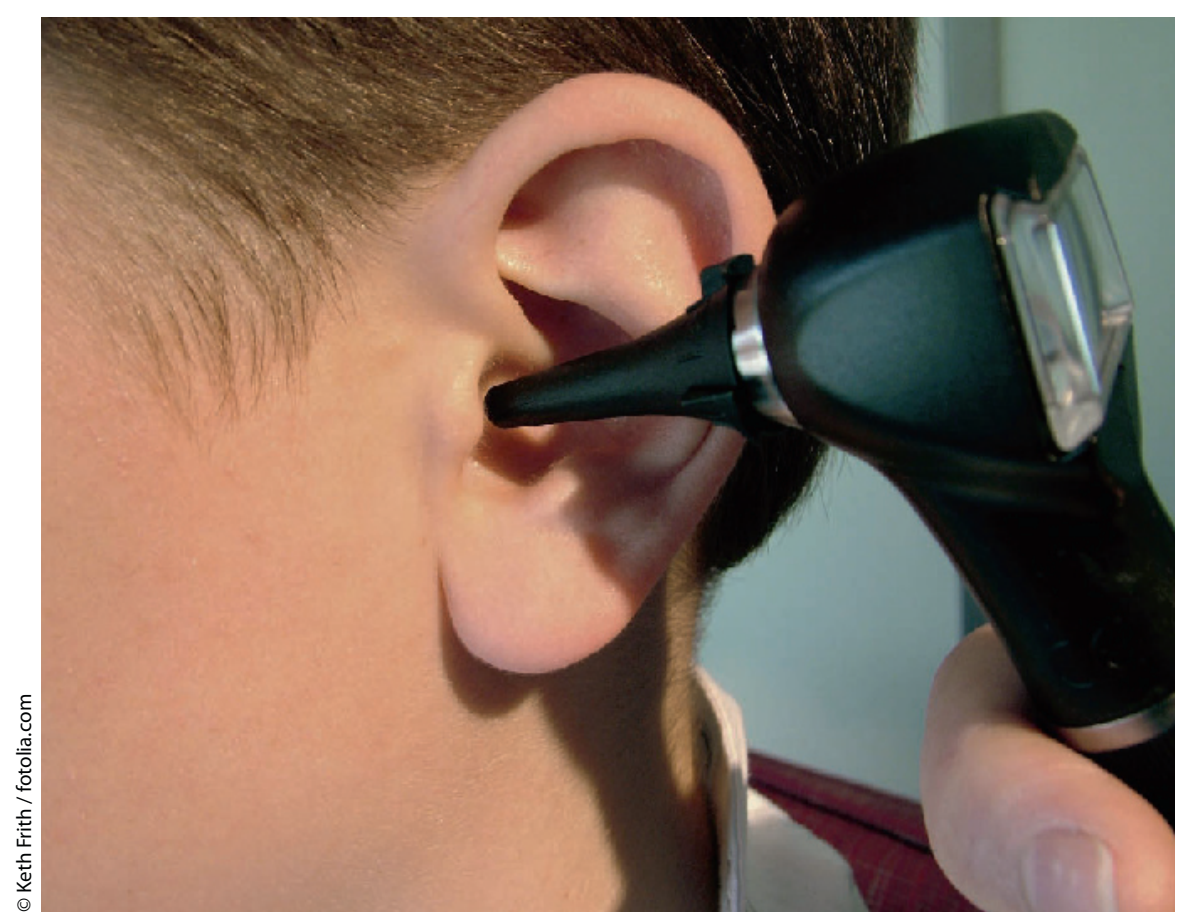

Zur Beseitigung der Erreger bei einer Otitis externa empfehlen die HNO-Leitlinien eine antibiotische Lokaltherapie.
Cetuximab im klinischen Alltag etabliert

Der monoklonale lgG1-Antikörper Cetuximab (Erbitux ${ }^{\oplus}$ ) ist die bisher einzige zugelassene zielgerichtete Therapieoption für die Behandlung von Plattenepithelkarzinomen im Kopf-HalsBereich. Wie im Rahmen eines interdisziplinären Satellitensymposiums beim ICHNO (3rd International Conference on Innovative Approaches in Head and Neck Oncology) diskutiert wurde, gilt der Antikörper heute in Kombination mit einer platinbasierten Chemotherapie als Therapiestandard für die Erstlinientherapie von rezidivierten und/ oder metastasierten Kopf-Hals-Tumoren. Die Experten zeigten sich darüber hinaus davon überzeugt, dass Cetuximab in Kombination mit einer Radiotherapie bei Patienten mit lokal fortgeschrittenem, nicht operablem Tumor eine sehr gute Alternative zur kombinierten Radiochemotherapie ist.

Nach Informationen von Merck Serono

\section{Umfangreiches Programm an Studien in der Allergologie}

Novartis Pharma hat gemeinsam mit spanischen Partner Leti Pharma ein umfangreiches Programm an Zulassungsstudien geplant. Damit kommen die Unternehmen den Anforderungen der Therapieallergeneverordnung von 2008, die eine Ausdehnung der Vorschriften zur Zulassung auf Therapieallergene vorsieht, in vollem Umfang nach.,"Wir begrüßen diesen Vorstoß des Gesetzgebers, einheitliche Standards für Therapieallergene zu fordern", so Dr. Dirk Kosche, Vorsitzender der Geschäftsführung der Novartis Pharma GmbH. Die beiden Unternehmen wollen die Forschungsanstrengungen im Bereich Allergologie noch weiter intensivieren und in den nächsten Jahren insgesamt mehr als 100 Millionen Euro in klinische Studien investieren. Konkret geplant sind eine Reihe klinischer Studien, um für alle relevanten Allergenextrakte und deren Mischungen der Präparate Depigoid $^{\circledR}$ für die perenniale subkutane Therapie, Depiquick ${ }^{\circledast}$ für die subkutane Kurzzeit-Immuntherapie und TOL SL plus für die sublinguale Therapie die Zulassung zu erlangen.

Nach Informationen von Novartis Pharma 